\title{
OPEN Loss of $\alpha$ klotho causes reduced motor ability and short lifespan in zebrafish
}

\author{
Yurie Ogura, Ryoji Kaneko, Kota Ujibe, Yuma Wakamatsu \& Hiromi Hirata
}

The klotho gene encodes a transmembrane protein $\alpha$ Klotho that interacts with a fibroblast growth factor (FGF) receptor in renal tubular epithelial cells and functions as a co-receptor for FGF23, which is an osteocytes-derived hormone. This bone-to-kidney signal promotes urinary phosphate excretion. Interestingly, $\alpha$ Klotho knockout mice show an accelerated aging and a shortened life span. Similarly, C. elegans lacking the aklotho homologue showed a short life span. However, the physiological basis of aging-related function of $\alpha$ klotho remain unclear. The $\alpha$ klotho-deficient vertebrate animals other than mice have been awaited as an alternative model of premature aging. We here employed zebrafish in our study and revealed that aklotho mutant zebrafish appeared to be normal at 3 months postfertilization (mpf) but eventually underwent premature death by $9 \mathrm{mpf}$, while normal zebrafish is known to survive for 42 months. We also assessed the motor ability of zebrafish in a forced swimming assay and found that $\alpha$ klotho mutant zebrafish displayed reduced swimming performance before their survival declined. A recent study also reported a similar finding that $\alpha$ klotho-deficient zebrafish exhibited a short life span and reduced spontaneous movements. Taken together, these results suggest that $\alpha$ Klotho mutant zebrafish show premature aging and are useful to investigate aging in vertebrates.

Senescence is a process of cell cycle arrest, while aging is a process of gradual deterioration of tissue functions. In human aging, a number of physiological decline of tissue functions occur over time, accompanying the increase of skin wrinkling, soft tissue calcification, neural degeneration, muscle weakness and motor deterioration ${ }^{1}$. Numerous aging-related disorders and premature aging diseases have been pathologically identified in human. Although it has been suggested that aging is triggered by an accumulation of DNA/cell damage or by a genetic limitation of cell proliferation, the physiological basis and causes of aging are still largely unknown ${ }^{1}$. To investigate aging-related genes and to fight against aging, several animal models such as mice, fruit flies and C. elegans have been used to assay longevity and premature death ${ }^{2-5}$. Zebrafish (Danio rerio), which is an emerging alternative vertebrate model, has also been used to study the progressive deterioration of biological functions in aging ${ }^{6,7}$.

The klotho $(k l)$ gene was originally identified as an aging-suppressor in mutant mice that showed an accelerated aging and a short lifespan ${ }^{8}$. The $k l$ gene encodes a single-pass transmembrane protein aklotho and is predominantly expressed in the distal convoluted tubules. The aklotho protein binds to fibroblast growth factor (FGF) receptors and functions as a co-receptor for FGF23, which is secreted from osteocytes in bone ${ }^{9}$. This bone-to-kidney signal is activated by an increase of serum phosphorus, promoting phosphate discharge from blood to urine ${ }^{10}$. The aklotho-deficient mice as well as FGF23 knockout mice exhibited an increase of blood phosphate that resulted in arteriosclerosis and vascular calcification ${ }^{11,12}$. In addition to these blood vessel disorders, multiple aging phenotypes such as skin atrophy, auditory disturbance, osteopenia, sarcopenia and premature death has been reported in these mutant mice ${ }^{13}$. Conversely, transgenic mice that overexpress aklotho displayed longevity compared to normal mice ${ }^{14}$. Thus, one important contributing factor to aklotho-mediated suppression of aging appears to be the regulation of phosphate levels. But how phosphate homeostasis determines the life span remains unclear ${ }^{15}$. The aklotho-deficient vertebrate animals other than mice would contribute toward understanding aging.

Here, we employed zebrafish in our aklotho study and found that aklotho mutant zebrafish (Y306X) showed a shortened lifespan. A recent study reported the same finding that aklotho-deficient zebrafish (S179frameshift) underwent a premature death ${ }^{16}$. We also assessed swimming performance of zebrafish in a forced swimming assay and demonstrated that aklotho mutant zebrafish display a reduced swimming ability before their survival declined. 
a

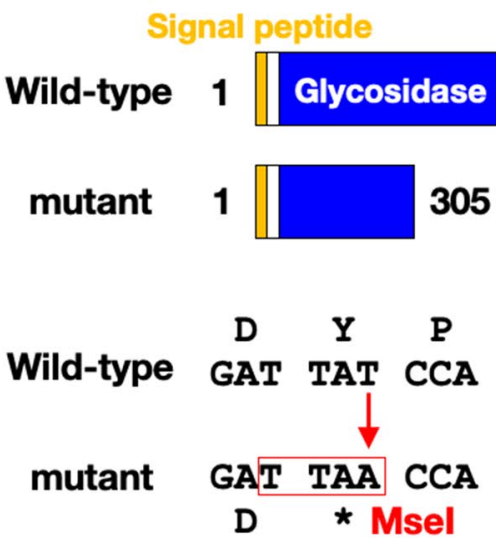

C

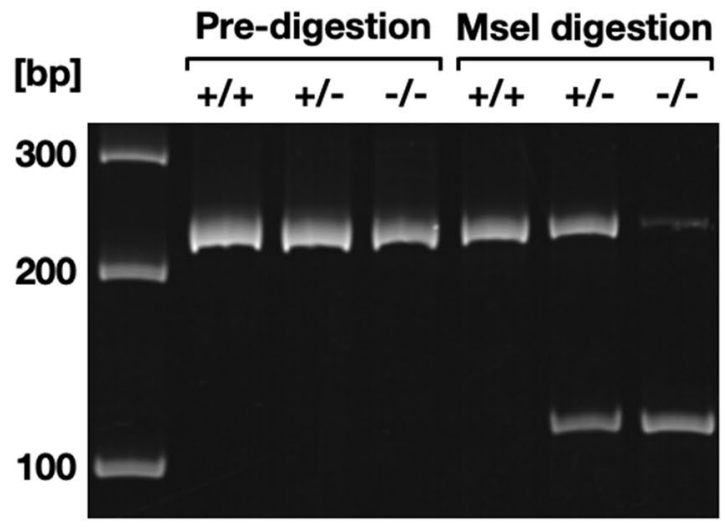

Figure 1. A nonsense mutation of zebrafish $k l$ gene. (a) The aklotho protein has a signal peptide, two glycosidase domains and a transmembrane domain. A nonsense mutation caused a truncation in the first glycosidase domain. (b) The T-to-A mutation generated an MseI restriction enzyme site. (c) The size of the genomic PCR products was $240 \mathrm{bp}$. The PCR products amplified from mutant alleles were digested by MseI to generate two 120 -bp fragments.

\section{Results}

Zebrafish klotho gene and klotho mutation. To study aklotho in zebrafish, we first retrieved klotho gene information of zebrafish and the other vertebrate animals from NCBI database. An amino acid alignment of the aklotho showed that aklotho protein is conserved among vertebrates from zebrafish to human, especially in two glycosidase domains (Supplemental Fig. 1). Although amino acid residues of the transmembrane domain at the C-terminus of the aklotho appeared to be less conserved in chicken, frog and zebrafish, online prediction tools of the protein secondary structure such as PredictProtein and SOSUI suggested that this region is a putative membrane-spanning domain in these non-mammalian animals, verifying that aklotho is overall conserved among vertebrates.

To investigate the physiological function of aklotho in zebrafish, we next obtained an aklotho mutant allele

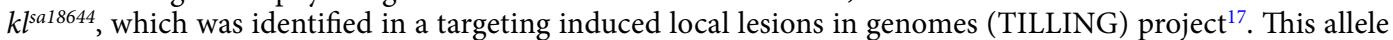
harbors a $\mathrm{T}$ to A base substitution that generates a premature nonsense codon (Y306X) in the middle of the first glycosidase domain (Fig. 1a). This mutation also generated an MseI restriction site that enabled genotyping by a restriction enzyme digestion of the genomic PCR products (Fig. 1b,c).

Zebrafish klotho mutants exhibit short life spans. By crossing heterozygous $k l$ mutant carrier fish, we obtained homozygous $k l$ mutants $\left(k l^{-1-}\right)$. The $k l^{l^{--}}$embryos showed no apparent defects and grew up to become adults. The ratio of $\mathrm{kl}^{-1-}$ mutants in a progeny of a heterozygous carrier cross was about one quarter $(23 \%, 23 / 100)$ at 4 months postfertilization (mpf), indicating that the loss of $k l$ does not affect development or survival of zebrafish in both females and males until they become young adults (Fig. 2a-d) as reported recently ${ }^{16}$. Although the skin of the previously reported $k l$-deficient zebrafish appeared to be pale at $5 \mathrm{mpf}^{16}$, we did not see apparent reduction of the skin tone in $\mathrm{kl}^{-/-}$compared to $\mathrm{kl}^{+/+}$fish at any age. Intriguingly, we noticed that some mutant female displayed protruding eye at $5 \mathrm{mpf}(30 \%, 6 / 20)$ just like a telescope goldfish (Fig. 2e-1). This malformation of the eye was not seen in mutant male fish $(\mathrm{n}=30)$. We also recapitulated that our $\mathrm{kl}^{-1-}$ fish become thinner after 5 months of age and die within 9 months (Fig. $2 \mathrm{~m}-\mathrm{p}$ ). To detail the short life span of $\mathrm{kl}^{-1-}$ zebrafish, we monitored the survival of our $\mathrm{kl}^{+/+}$and $\mathrm{kl}^{-{ }^{--}}$fish from $2 \mathrm{mpf}$ (Fig. 2q). We found that all of the $k l^{l^{-1}}$ fish $(\mathrm{n}=28)$ died between 4 and $9 \mathrm{mpf}$, whereas all $\mathrm{kl}^{l^{++}}$fish $(\mathrm{n}=32)$ kept living after $9 \mathrm{mpf}$. It has been reported that the mean life span of zebrafish is 42 months $^{18}$. These results indicate that $\mathrm{kl}^{-1-}$ zebrafish undergo premature aging and death.

Zebrafish klotho mutants show reduced motor ability. Since aging affects the motor system ${ }^{19}$, we addressed whether motor ability is impaired in $\mathrm{kl}^{-1-}$ zebrafish. As swimming ability of adult zebrafish is governed by the body size especially by the caudal fin length ${ }^{20}$, we began with a physical measurement. We measured the standard length and caudal fin length, which are defined as the length from the head to the root of the caudal fin and from the root of the caudal fin to the edge of the fin, respectively ${ }^{21}$. Both standard length and caudal fin length of $\mathrm{kl}^{+++}$and $\mathrm{kl}^{-/-}$fish were comparable in both females and males at $3 \mathrm{mpf}$ and $4 \mathrm{mpf}$. These data confirm that $k l^{-/-}$fish are indistinguishable from $k l^{+++}$fish by appearance at both $3 \mathrm{mpf}$ and $4 \mathrm{mpf}$.

To quantify the swimming performance of adult zebrafish, we then employed a swimmill, which is a treadmill for aquatic animals ${ }^{20}$. A zebrafish was put in a swimming chamber, where water flow is generated by a voltagecontrolled spinning propeller. Since fish swim against current to maintain the position in the water flow, zebrafish in the chamber swim at the water velocity. The water velocity was initially set to $10 \mathrm{~cm} / \mathrm{s}$ for 1 min and successively set to $15 \mathrm{~cm} / \mathrm{s}$ for $1 \mathrm{~min}$. Eventually, the water velocity increased $1 \mathrm{~cm} / \mathrm{s}$ every $1 \mathrm{~min}$ and the water velocity at when zebrafish could no longer keep swimming was defined as the critical swimming speed $\left(U_{\text {crit }}\right)$ as described previously ${ }^{22}$. At $3 \mathrm{mpf}$, the $U_{\text {crit }}$ of $k l^{-l-}$ zebrafish (female $22.2 \pm 2.9 \mathrm{~cm} / \mathrm{s}, \mathrm{n}=4$; male $22.0 \pm 2.8 \mathrm{~cm} / \mathrm{s}, \mathrm{n}=3$; Fig. 3) 


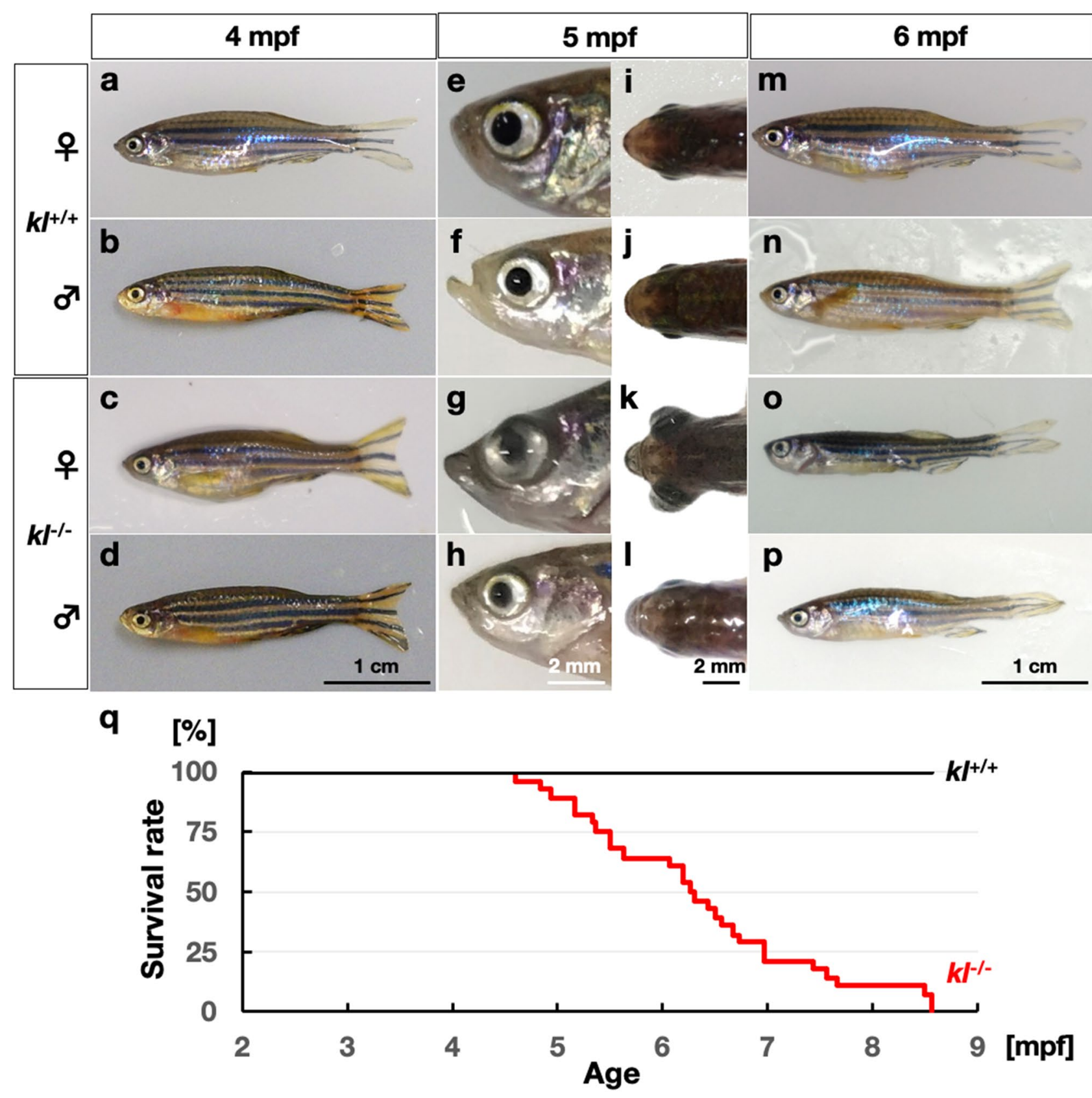

Figure 2. Zebrafish $k l$ mutants become thinner and undergo premature death. (a-d) The $k l^{+/+}$female (a), $k l^{+/+}$ male (b), $k l^{-1-}$ female (c) and $k l^{-1-}$ male (d) zebrafish at $4 \mathrm{mpf}$. The $k l^{-1-}$ fish showed no morphological defects at this stage. (e-l) Lateral and dorsal views of head in $k l^{+++}$female (e,i), $k l^{+++}$male (f,j), $k l^{-1-}$ female (g,k) and $k l^{-/-}$ male $(\mathbf{h}, \mathbf{l})$ at $5 \mathrm{mpf}$. Note that the eyeball is protruding to the outside in the $k l^{-/-}$female. $(\mathbf{m}-\mathbf{p})$ The $k l^{+/+}$female $(\mathbf{m}), k l^{l_{+}}$male (n), $k l^{l_{-1-}}$ female (o) and $k l^{l^{-1}}$ male (p) zebrafish at 6 mpf. The $k l^{l^{-1}}$ fish became skinny. (q) The $k l^{+++}$fish $(\mathrm{n}=32)$ survived over $9 \mathrm{mpf}$. All of the $k l^{-I-}$ fish $(\mathrm{n}=28)$ underwent premature death before $9 \mathrm{mpf}$.

were comparable to those of $k l^{+/+}$fish (female $24.0 \pm 3.3 \mathrm{~cm} / \mathrm{s}, \mathrm{n}=3, P=0.70$; male $24.0 \pm 2.2 \mathrm{~cm} / \mathrm{s}, \mathrm{n}=4 ; P=0.59$ ). At $4 \mathrm{mpf}$, however, the $U_{\text {crit }}$ of $k l^{-1-}$ zebrafish (female $18.6 \pm 0.8 \mathrm{~cm} / \mathrm{s}, \mathrm{n}=9$; male $15.0 \pm 2.5 \mathrm{~cm} / \mathrm{s}, \mathrm{n}=3$ ) were significantly lower than those of $\mathrm{kl}^{l^{++}}$fish (female $26.4 \pm 0.6 \mathrm{~cm} / \mathrm{s}, \mathrm{n}=13, P<0.001$; male $27.4 \pm 1.6 \mathrm{~cm} / \mathrm{s}, \mathrm{n}=6$; $P<0.01)$. These results demonstrate that the swimming performance of $k l^{-l-}$ fish starts to decline between 3 and $4 \mathrm{mpf}$ when they do not exhibit premature death.

\section{Discussion}

In this study, we investigated a nonsense mutant allele of $k l$ in zebrafish and found that $k l^{-1-}$ fish showed a shortened life span. Recently, Singh and his colleagues have reported the other $k l$ mutant zebrafish allele, which harbors a CRISPR-mediated 5-bp deletion and thus creates a frameshift in the first glycosidase domain ${ }^{16}$. Although age-related frailty and premature death in our $\mathrm{kl}^{-1-}$ fish were recapitulations of a recent report, we uniquely 


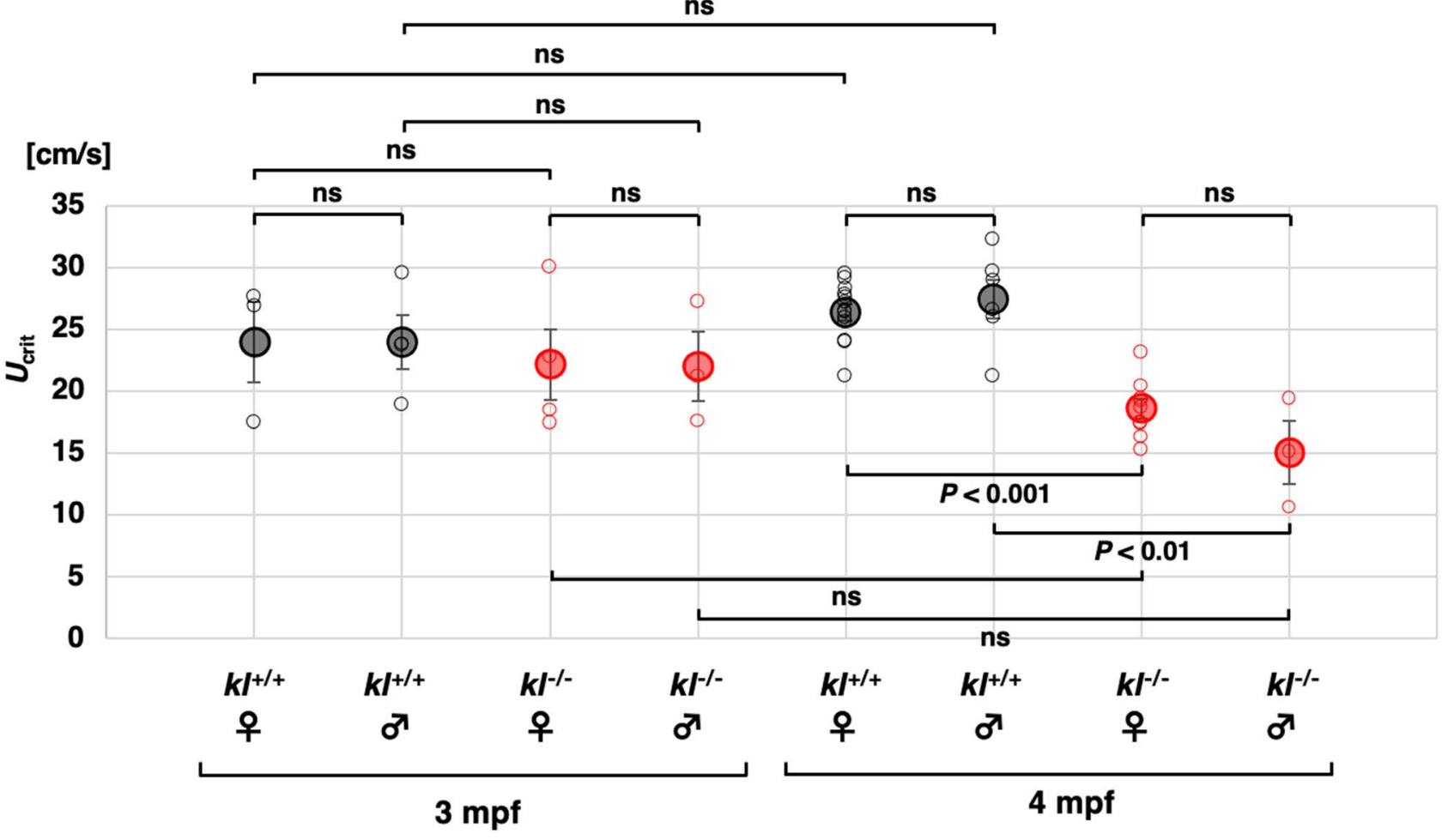

Figure 3. Swimming performance in $k l$ mutants declines by $4 \mathrm{mpf}$. The critical swimming speed $\left(U_{\text {crit }}\right)$ of $3 \mathrm{mpf}$ and $4 \mathrm{mpf}$ zebrafish were measured using a swimmill. The $k l^{+/+}$female $(\mathrm{n}=3), k l^{+/+}$male $(\mathrm{n}=4), k l^{-1-}$ female $(\mathrm{n}=4)$ and $k l^{l^{--}}$male $(\mathrm{n}=3)$ were assayed at $3 \mathrm{mpf}$. The $k l^{+/+}$female $(\mathrm{n}=13), k l^{+/+}$male $(\mathrm{n}=9), k l^{-/-}$female $(\mathrm{n}=6)$ and $k l^{-1-}$ male $(\mathrm{n}=3)$ were assayed at $4 \mathrm{mpf}$. Open black $\left(k l^{l^{++}}\right)$and red $\left(k l^{-l-}\right)$ circles indicate $U_{\text {crit }}$ of individual samples. Closed black $\left(k l^{+++}\right)$and red $\left(k l^{-l-}\right)$ circles with error bars indicate mean \pm sem.

examined swimming performance of $k l^{-/-}$fish using a swimmill and demonstrated that $k l^{-/-}$fish initially exhibited comparable motor performance with $\mathrm{kl}^{+/+}$fish at $3 \mathrm{mpf}$ and eventually showed a compromised swimming at $4 \mathrm{mpf}$ before they become thinner and undergo premature death at 5-8 mpf. Taken together, we conclude that aklotho mutant zebrafish show premature aging phenotypes and that aklotho-deficient zebrafish can be an animal model of progeria.

Conservation of the $\alpha$ klotho in vertebrates. While invertebrate animals use calcium carbohydrate for exoskeleton, vertebrate animals utilize calcium phosphate for endoskeleton but with a risk of phosphate toxicity ${ }^{23}$. Since aklotho plays an essential role in discharging excess phosphate from blood to prevent unwanted ectopic calcification, it is reasonable that aklotho function is conserved among vertebrates ${ }^{24}$. Our amino acid alignment revealed that aklotho proteins have two putative glycosidase domains that are highly conserved among vertebrates. These domains share the highest homology with lactase, which hydrolyses lactose to produce galactose and glucose ${ }^{25,26}$. Interestingly however, the catalytic glutamate residues in both glycosidase domains in aklotho were substituted to the other residues; i.e. N239 and S872 in human, N241 and A874 in mice and N208 and A849 in zebrafish. Instead of losing glycosidase activities, these domains appeared to acquire an affinity to a calciotropic hormone FGF23, enabling aklotho to function as a co-receptor of FGF23 for the excretion of phosphate from renal tubules. These amino acid substitution might have been critical for aklotho function in vertebrates. An aklotho homologue gene (klo1) was also found in C. elegans, and klo1-deficient worms showed short life spans ${ }^{27,28}$. The aklotho protein in C. elegans has only one glycosidase domain with its catalytic residue intriguingly maintained as glutamate, potentially possessing the glycosidase activity. But how aklotho-mediated suppression of aging takes place in C. elegans remains unsolved. Our $k l$ mutant allele harbored a nonsense mutation in the first putative glycosidase domain, while the other allele reported by Singh et al. carried a frameshift mutation in the same glycosidase domain ${ }^{16}$. Both of these mutant alleles appear to disrupt the putative glycosidase domain and eliminate the transmembrane domain at the $\mathrm{C}$-terminus and thus are likely unfunctional as a co-receptor for FGF23.

Telescope eye phenotype in $\mathbf{k l}^{-l-}$ female zebrafish. The telescope eye goldfish strain was established 300 years ago and maintained in East Asia $^{29}$. This developmental malformation of the eye is inherited to both female and male offspring in a recessive manner. A recent study revealed that nonsense mutations or a retrotransposon insertion in goldfish $\operatorname{lrp} 2 a l$ gene, which encodes a low density lipoprotein receptor-related protein, was responsible for the telescope eye $\mathrm{e}^{30}$. Likewise, the $\operatorname{lrp} 2 a$ mutation in zebrafish caused the protruding eye phenotype, which is often referred to as bugeye, in both female and male individuals with a variable penetra- 
tion rate $31-33$. In our zebrafish $\mathrm{kl}^{-/-}$case, telescope eye phenotype was seen in $30 \%$ of $\mathrm{kl}^{-l_{-}}$female but not in any $\mathrm{kl}^{-1-}$ male. Similarly, fgf23-deficient zebrafish exhibited the protruding eye only in the female ${ }^{16}$. Collectively, the aklotho-FGF23 signals may potentially regulate the expression or function of $\operatorname{lrp} 2 a$ in cooperation with the female-specific endocrine regulation, thereby affecting the eye development in female.

Decline of motor performance in $\mathbf{k l}^{-l_{-}^{-}}$zebrafish. Previous in situ hybridization analyses revealed that $k l$ gene is expressed by the brain, pancreas, liver and pronephros in embryonic and larval zebrafish and by the liver and mesonephric kidney tubules in adult zebrafish ${ }^{34}$. The $\mathrm{kl}^{-1-}$ zebrafish did not show any apparent defects during development and in young adults. But they eventually showed the decline of motor ability at $4 \mathrm{mpf}$, became thinner at $6 \mathrm{mpf}$ and underwent premature death by $9 \mathrm{mpf}$. Although mutant phenotypes in zebrafish larvae can be generally rescued by mRNA injection-mediated overexpression of the gene product, this does not work in $\mathrm{kl}^{-1-}$ zebrafish, because all of the defects in $\mathrm{kl}^{-1-}$ were adult onset.

Since $k l$ gene is expressed only by the liver and kidney in adult zebrafish ${ }^{34}$, the motor impairment, which is attributable to the neuronal dysfunction, muscle weakness or cardiovascular defects, could be a secondary phenotype caused through a perturbation of the endocrine system. Our future histological and physiological studies of neurons and muscles as well as metabolomic analyses at 3-4 mpf will clarify the basis of the motor deterioration. In our forced swimming assay, the decline of the motor ability was evaluated by the maximum swimming speed $U_{\text {crit }}$. It has been reported that the reduction of $U_{\text {crit }}$ accompanies with aging in zebrafish ${ }^{35}$, implying that motor deterioration in $\mathrm{kl}^{-/-}$fish is a premature aging phenotype. Taken together, the decline of motor performance is likely one of the earliest physiological phenotype of premature aging in $k l^{-1-}$ zebrafish.

Pathology of $\alpha$ klotho. Kuro-o and his colleagues have demonstrated that aklotho mutant mice showed cellular senescence such as the upregulation of a cyclin-dependent kinase inhibitor p21 and cell cycle arrest as well as age-related disorders including the ectopic calcification and hypokinesis ${ }^{8}$. The life span of the mutant mice was approximately 60 days with no mice surviving over 100 days. A population-based association study suggested that a SNP that generates an F352V missense variant in human aklotho is implicated in a short life $\operatorname{span}^{36}$. In addition, a 13-year-old girl who carries a homozygous H193R missense variant in aklotho showed premature tumoral calcinosis with dural and carotid artery calcifications ${ }^{37}$. Thus, physiological symptoms linked to aklotho mutations appear to be common in vertebrates. Although pathological studies of aklotho deficiency has only been done in human, mice and C. elegans until recently, the latest publication of aklotho mutant zebrafish ${ }^{16}$ and our current study demonstrated pathologically-relevant progressive motor deterioration and premature death in zebrafish $k l$ mutants. Collectively, zebrafish aklotho mutants provide an alternative animal model to study cellular senescence, aging and progeria in vertebrates.

\section{Materials and methods}

Animals. Zebrafish (Danio rerio) were reared and maintained in $1.7 \mathrm{~L}$ tanks in a recirculating Meito System (Meito System) under a $14 \mathrm{~h}$ light and $10 \mathrm{~h}$ dark photoperiod according to the standard protocol ${ }^{38}$. Larvae were fed paramecia and Gemma Micro ZF 75 (Funakoshi) twice a day from 5 days postfertilization to 1 mpf. Juvenile fish (1-3 mpf) were fed brine shrimp (Tokai Guppy) and Gemma Micro ZF 75 twice a day. Adults fish (3 mpf-) were fed brine shrimp and Otohime B2 (Marubeni Nissin Feed) twice a day. Zebrafish aklotho mutant line $\left(k^{\text {sal8644 }}\right)$ and wild-type $\mathrm{AB}$ line, the latter for the line maintenance, was purchased from Zebrafish International Resource Center (https://zebrafish.org/home/guide.php).

Genotyping. The missense region of $k l$ gene was amplified by genomic PCR using KAPA Taq Extra PCR Kit (Kapa Biosystems) in ProFlex PCR System (Thermo Fisher Scientific). Following program was used for amplification: $94^{\circ} \mathrm{C} 2 \mathrm{~min} ; 94^{\circ} \mathrm{C} 10 \mathrm{~s}, 63{ }^{\circ} \mathrm{C} 20 \mathrm{~s}, 72^{\circ} \mathrm{C} 30 \mathrm{~s}, 35$ cycles; $72^{\circ} \mathrm{C} 1 \mathrm{~min} ; 4^{\circ} \mathrm{C}$ forever. Following primers were used; $k l$ genotyping forward: CTCTGGGATCTCACTGGATC; $k l$ genotyping reverse: AACTAAGAGCAG GTCCATGAGAC. PCR products were digested with MseI restriction enzyme (Takara) and separated by $15 \%$ polyacrylamide gel electrophoresis at $300 \mathrm{~V}$ for $90 \mathrm{~min}$ as described previously ${ }^{39}$. The gel images were captured using the Printgraph AE-6933FXCF (Atto).

Image capture. Zebrafish were anesthetized in 0.004\% Tricaine (MS-222, Sigma-Aldrich) for 1 min. Images of zebrafish were captured using a digital camera SONY $\alpha 5000$.

Survival. Adult zebrafish obtained by a cross of $\mathrm{kl}^{+/-}$fish were maintained in the regular care. Genotyping was done at $2 \mathrm{mpf}$. Zebrafish $\left(\mathrm{kl}^{+/+}\right.$and $\left.\mathrm{kl}^{-/-}\right)$that kept floating at water surface or sinking at the bottom without swimming for $1 \mathrm{~min}$ were judged as reaching the end point and subjected to euthanasia and genotyping. Number of zebrafish reaching the end point was counted everyday until all of the $\mathrm{kl}^{-1-}$ fish died.

Swimmill analysis. Motor ability of zebrafish was quantified using a swimmill system Swim tunnel respirometer $170 \mathrm{ml}$ (Loligo System) as described previously ${ }^{20}$. In brief, an adult zebrafish, which was kept unfed for $24 \mathrm{~h}$, was put under a propeller-driven water flow in a chamber, and fish was compelled to swim in the water flow. The water velocity increased $1 \mathrm{~cm} / \mathrm{s}$ every $1 \mathrm{~min}$ after initial warming up of $10 \mathrm{~cm} / \mathrm{s}$ flow for $1 \mathrm{~min}$ and successive $15 \mathrm{~cm} / \mathrm{s}$ flow for $1 \mathrm{~min}$. Zebrafish swam at the speed of water flow until the water velocity reach the maximum swimming capability of the fish. The water velocity at when zebrafish can no longer keep swimming was defined as critical swimming speed $U_{\text {crit }}$. The standard and caudal fin lengths were measured by analyzing frames of swimming movies as described previously ${ }^{20}$. 
Statistics. The quantitative data were given as mean \pm sem. All error bars in graphs indicate the sem. The sample numbers are shown in Figure legends. The quantitative data were tested for normality by Shapiro-Wilk test $(\mathrm{P}<0.05)$. Statistical significance was determined by multiple comparison tests using the analysis of variance (ANOVA).

Ethics statement. This study was approved by Animal Care and Use Committee of Aoyama Gakuin University (A9/2020) and carried out according to the Aoyama Gakuin University Animal Care and Use Guideline.

Received: 7 December 2020; Accepted: 28 June 2021

Published online: 23 July 2021

\section{References}

1. Lopez-Otin, C., Blasco, M. A., Partridge, L., Serrano, M. \& Kroemer, G. The hallmarks of aging. Cell 153, 1194-1217. https://doi. org/10.1016/j.cell.2013.05.039 (2013).

2. Clancy, D. J. et al. Extension of life-span by loss of CHICO, a Drosophila insulin receptor substrate protein. Science 292, 104-106. https://doi.org/10.1126/science.1057991 (2001).

3. Festing, M. F. \& Blackmore, D. K. Life span of specified-pathogen-free (MRC category 4) mice and rats. Lab Anim. 5, 179-192. https://doi.org/10.1258/002367771781006564 (1971).

4. Kenyon, C., Chang, J., Gensch, E., Rudner, A. \& Tabtiang, R. A. C. elegans mutant that lives twice as long as wild type. Nature 366, 461-464. https://doi.org/10.1038/366461a0 (1993).

5. Tatar, M. et al. A mutant Drosophila insulin receptor homolog that extends life-span and impairs neuroendocrine function. Science 292, 107-110. https://doi.org/10.1126/science.1057987 (2001).

6. Gerhard, G. S. \& Cheng, K. C. A call to fins! Zebrafish as a gerontological model. Aging Cell 1, 104-111. https://doi.org/10.1046/j. 1474-9728.2002.00012.x (2002).

7. Grunwald, D. J. \& Eisen, J. S. Headwaters of the zebrafish-Emergence of a new model vertebrate. Nat. Rev. Genet. 3, 717-724. https://doi.org/10.1038/nrg892 (2002).

8. Kuro-o, M. et al. Mutation of the mouse klotho gene leads to a syndrome resembling ageing. Nature 390, 45-51. https://doi.org/ $10.1038 / 36285$ (1997).

9. Kurosu, H. et al. Regulation of fibroblast growth factor-23 signaling by klotho. J. Biol. Chem. 281, 6120-6123. https://doi.org/10. 1074/jbc.C500457200 (2006).

10. Kuro-o, M. Klotho, phosphate and FGF-23 in ageing and disturbed mineral metabolism. Nat. Rev. Nephrol. 9, 650-660. https:// doi.org/10.1038/nrneph.2013.111 (2013).

11. Shimada, T. et al. Targeted ablation of Fgf23 demonstrates an essential physiological role of FGF23 in phosphate and vitamin D metabolism. J. Clin. Investig. 113, 561-568. https://doi.org/10.1172/JCI19081 (2004).

12. Sitara, D. et al. Homozygous ablation of fibroblast growth factor-23 results in hyperphosphatemia and impaired skeletogenesis, and reverses hypophosphatemia in Phex-deficient mice. Matrix Biol. 23, 421-432. https://doi.org/10.1016/j.matbio.2004.09.007 (2004).

13. Edmonston, D. \& Wolf, M. FGF23 at the crossroads of phosphate, iron economy and erythropoiesis. Nat. Rev. Nephrol. 16, 7-19. https://doi.org/10.1038/s41581-019-0189-5 (2020).

14. Kurosu, H. et al. Suppression of aging in mice by the hormone Klotho. Science 309, 1829-1833. https://doi.org/10.1126/science. 1112766 (2005).

15. Kuro-o, M. The Klotho proteins in health and disease. Nat. Rev. Nephrol. 15, 27-44. https://doi.org/10.1038/s41581-018-0078-3 (2019).

16. Singh, A. P. et al. alphaKlotho regulates age-associated vascular calcification and lifespan in zebrafish. Cell Rep. 28, 2767-2776 e2765. https://doi.org/10.1016/j.celrep.2019.08.013 (2019).

17. Kettleborough, R. N. et al. A systematic genome-wide analysis of zebrafish protein-coding gene function. Nature 496, $494-497$. https://doi.org/10.1038/nature11992 (2013).

18. Gerhard, G. S. et al. Life spans and senescent phenotypes in two strains of Zebrafish (Danio rerio). Exp. Gerontol. 37, $1055-1068$. https://doi.org/10.1016/s0531-5565(02)00088-8 (2002).

19. Brunet, A. Old and new models for the study of human ageing. Nat. Rev. Mol. Cell Biol. https://doi.org/10.1038/s41580-020-0266-4 (2020).

20. Wakamatsu, Y., Ogino, K. \& Hirata, H. Swimming capability of zebrafish is governed by water temperature, caudal fin length and genetic background. Sci. Rep. 9, 16307. https://doi.org/10.1038/s41598-019-52592-w (2019).

21. Plaut, I. Effects of fin size on swimming performance, swimming behaviour and routine activity of zebrafish Danio rerio. J. Exp. Biol. 203, 813-820 (2000).

22. Brett, J. R. The respiratory metabolism and swimming performance of young sockeye salmon. J. Fish. Res. Board Can. https://doi. org/10.1139/f64-103 (1964).

23. Stenvinkel, P. et al. Novel treatment strategies for chronic kidney disease: Insights from the animal kingdom. Nat. Rev. Nephrol. 14, 265-284. https://doi.org/10.1038/nrneph.2017.169 (2018).

24. Kuro, O. M. \& Moe, O. W. FGF23-alphaKlotho as a paradigm for a kidney-bone network. Bone 100, 4-18. https://doi.org/10.1016/j. bone.2016.11.013 (2017).

25. Ito, S., Fujimori, T., Hayashizaki, Y. \& Nabeshima, Y. Identification of a novel mouse membrane-bound family 1 glycosidase-like protein, which carries an atypical active site structure. Biochim. Biophys. Acta 1576, 341-345. https://doi.org/10.1016/s01674781(02)00281-6 (2002).

26. Sugano, Y. \& Lardelli, M. Identification and expression analysis of the zebrafish orthologue of Klotho. Dev. Genes Evol. 221, $179-186$. https://doi.org/10.1007/s00427-011-0367-3 (2011).

27. Chateau, M. T., Araiz, C., Descamps, S. \& Galas, S. Klotho interferes with a novel FGF-signalling pathway and insulin/Igf-like signalling to improve longevity and stress resistance in Caenorhabditis elegans. Aging (Albany NY) 2, 567-581. https://doi.org/10. 18632/aging.100195 (2010).

28. Polanska, U. M., Edwards, E., Fernig, D. G. \& Kinnunen, T. K. The cooperation of FGF receptor and Klotho is involved in excretory canal development and regulation of metabolic homeostasis in Caenorhabditis elegans. J. Biol. Chem. 286, 5657-5666. https://doi. org/10.1074/jbc.M110.173039 (2011).

29. Chen, S. C. A history of the domestication and the factors of the varietal formation of the common goldfish, Carassius auratus. Sci. Sin. 5, 287-321 (1956). 
30. Kon, T. et al. The genetic basis of morphological diversity in domesticated goldfish. Curr. Biol. 30, 2260-2274 e2266. https://doi. org/10.1016/j.cub.2020.04.034 (2020).

31. Sherpa, T., Hunter, S. S., Frey, R. A., Robison, B. D. \& Stenkamp, D. L. Retinal proliferation response in the buphthalmic zebrafish, bugeye. Exp. Eye Res. 93, 424-436. https://doi.org/10.1016/j.exer.2011.06.001 (2011).

32. Stujenske, J. M., Dowling, J. E. \& Emran, F. The bugeye mutant zebrafish exhibits visual deficits that arise with the onset of an enlarged eye phenotype. Investig. Ophthalmol. Vis. Sci. 52, 4200-4207. https://doi.org/10.1167/iovs.10-6434 (2011).

33. Veth, K. N. et al. Mutations in zebrafish $\operatorname{lrp} 2$ result in adult-onset ocular pathogenesis that models myopia and other risk factors for glaucoma. PLoS Genet. 7, e1001310. https://doi.org/10.1371/journal.pgen.1001310 (2011).

34. Mangos, S. et al. Expression of fgf23 and alphaklotho in developing embryonic tissues and adult kidney of the zebrafish, Danio rerio. Nephrol. Dial. Transplant. 27, 4314-4322. https://doi.org/10.1093/ndt/gfs335 (2012).

35. Gilbert, M. J. H., Zerulla, T. C. \& Tierney, K. B. Zebrafish (Danio rerio) as a model for the study of aging and exercise: Physical ability and trainability decrease with age. Exp. Gerontol. 50, 106-113. https://doi.org/10.1016/j.exger.2013.11.013 (2014).

36. Arking, D. E. et al. Association of human aging with a functional variant of klotho. Proc. Natl. Acad. Sci. U.S.A. 99, 856-861. https:// doi.org/10.1073/pnas.022484299 (2002).

37. Ichikawa, S. et al. A homozygous missense mutation in human KLOTHO causes severe tumoral calcinosis. J. Clin. Investig. 117, 2684-2691. https://doi.org/10.1172/JCI31330 (2007).

38. Westerfield, M. The Zebrafish Book: A Guide for the Laboratory Use of Zebrafish (Danio rerio) 5th edn. (University of Oregon, 2007).

39. Ogino, K. et al. RING finger protein 121 facilitates the degradation and membrane localization of voltage-gated sodium channels. Proc. Natl. Acad. Sci. U.S.A. 112, 2859-2864. https://doi.org/10.1073/pnas.1414002112 (2015).

\section{Acknowledgements}

We thank Dr. Makoto Kashima (Aoyama Gakuin University) for fruitful discussion and Hirata Lab members for fish care. This work was supported by KAKENHI (Grant-in-Aid for Scientific Research B from the MEXT, Japan: 19H03329), the Takeda Science Foundation, the Naito Foundation.

\section{Author contributions}

H.H. designed research; Y.O., R.K., K.U. and Y.W. performed research and analyzed data; Y.O. and H.H. wrote the manuscript.

\section{Competing interests}

The authors declare no competing interests.

\section{Additional information}

Supplementary Information The online version contains supplementary material available at https://doi.org/ 10.1038/s41598-021-93909-y.

Correspondence and requests for materials should be addressed to H.H.

Reprints and permissions information is available at www.nature.com/reprints.

Publisher's note Springer Nature remains neutral with regard to jurisdictional claims in published maps and institutional affiliations.

(c) (i) Open Access This article is licensed under a Creative Commons Attribution 4.0 International License, which permits use, sharing, adaptation, distribution and reproduction in any medium or format, as long as you give appropriate credit to the original author(s) and the source, provide a link to the Creative Commons licence, and indicate if changes were made. The images or other third party material in this article are included in the article's Creative Commons licence, unless indicated otherwise in a credit line to the material. If material is not included in the article's Creative Commons licence and your intended use is not permitted by statutory regulation or exceeds the permitted use, you will need to obtain permission directly from the copyright holder. To view a copy of this licence, visit http://creativecommons.org/licenses/by/4.0/.

(C) The Author(s) 2021 